\title{
Simplified, minimally invasive, beating- heart technique for redo isolated tricuspid valve surgery
}

\author{
Shuyang $\mathrm{Lu}^{1+}$, Kai Song ${ }^{1+}$, Wangchao Yao ${ }^{1+}$, Limin Xia ${ }^{1}$, Lili Dong ${ }^{2}$, Yongxin Sun ${ }^{1}$, Tao Hong ${ }^{1}$, Shouguo Yang ${ }^{1 *}$ and \\ Chunsheng Wang ${ }^{1 *}$
}

\begin{abstract}
Background: Redo isolated tricuspid valve surgery is associated with a high morbidity and mortality, and its optimal timing remains controversial. Hence, here we reviewed the early and midterm results of simplified, minimally invasive, beating-heart technique for redo isolated tricuspid valve surgery in patients at high risk.

Methods: A total of 32 consecutive patients underwent a redo isolated tricuspid valve surgery using minimally invasive, beating-heart technique through a right lateral thoracotomy in our center between June 2016 and April 2020. The mean age of patients was $57.4 \pm 8.3$ years, and 18 patients (56.3\%) were women. The mean preoperative EuroSCORE was $7.8 \pm 1.4$ (range: 6-11). Follow-up was $87.1 \%$ complete, with a mean duration of $26.3 \pm 12.3$ months.

Results: Both in-hospital and 30-day mortalities were $3.1 \%$. Tricuspid valve replacement with bioprosthesis was performed in 30 patients (93.8\%), and the remaining two patients (6.2\%) underwent tricuspid repair (annuloplasty and leaflet reconstruction). The mean cardiopulmonary bypass time was $81.5 \pm 29.0 \mathrm{~min}$. The overall in-hospital duration and intensive care unit (ICU) times were $13.6 \pm 7.6$ days and $4.1 \pm 2.8$ days, respectively. Postoperative complications included prolonged ventilation in six patients (18.8\%), acute kidney injury in three patients (9.4\%), and neurologic event, wound infection, or permanent third-degree atrioventricular block, in one patient (3.1\%) each. A total of 96.9\% patients were discharged uneventfully. Four patients were lost to follow-up; there were no midterm deaths in patients who were followed up.
\end{abstract}

Conclusions: Simplified, minimally invasive, beating-heart technique for redo tricuspid valve surgery is both feasible and safe, and the early and midterm results are excellent.

Keywords: Tricuspid valve surgery, Minimally invasive surgery, Redo

\section{Introduction}

Tricuspid valve disease has always been considered less clinically important than mitral and aortic valve pathology, and its optimal timing for surgical intervention remains controversial [1]. Many patients undergo concomitant

\footnotetext{
*Correspondence: yang.shouguo@zs-hospital.sh.cn; wang_cs@126.com †'Shuyang Lu, Kai Song and Wangchao Yao contributed equally to this work. 'Department of Cardiovascular Surgery, Zhongshan Hospital, Fudan University, The Shanghai Institute of Cardiovascular Diseases, No. 1609 Xietu Road, Xuhui District, Shanghai 200032, China

Full list of author information is available at the end of the article
}

tricuspid valve surgery at the time of mitral or aortic valve intervention, while isolated tricuspid valve surgery is rarely performed [2]. However, in a large number of cases, secondary isolated tricuspid valve regurgitation develops after previous cardiac operations, which is neglected and undertreated. Considering low pressure and low resistance of the right-sided cardiac valves, patients with tricuspid valve regurgitation usually do not have significant symptoms at an early stage; therefore, they are rarely referred for isolated surgical intervention of the tricuspid valve

(c) The Author(s). 2020 Open Access This article is licensed under a Creative Commons Attribution 4.0 International License, which permits use, sharing, adaptation, distribution and reproduction in any medium or format, as long as you give appropriate credit to the original author(s) and the source, provide a link to the Creative Commons licence, and indicate if changes were made. The images or other third party material in this article are included in the article's Creative Commons licence, unless indicated otherwise in a credit line to the material. If material is not included in the article's Creative Commons licence and your intended use is not permitted by statutory regulation or exceeds the permitted use, you will need to obtain permission directly from the copyright holder. To view a copy of this licence, visit http://creativecommons.org/licenses/by/4.0/. The Creative Commons Public Domain Dedication waiver (http://creativecommons.org/publicdomain/zero/1.0/) applies to the data made available in this article, unless otherwise stated in a credit line to the data. 
until right heart failure, uncontrolled ascites, or edema of lower extremities develop [3, 4]. In this context, isolated tricuspid valve surgery is often associated with a high morbidity and mortality [5-7].

Median sternotomy is the standard approach for redo surgery and in patients where the tricuspid valve must be treated. However, as the development of minimally invasive surgery for atrioventricular valves, atrial septal defects, and atrial fibrillation, this technique has also been used in redo surgeries $[8,9]$. Some centers have reported their experience with minimally invasive tricuspid valve surgery [10-12], but the details of surgical techniques (such as arterial inflow, venous drainage, aortic cross-clamping or not, and cooling temperature) varied among different centers. One of the questions requiring attention is whether redo tricuspid valve surgery can be successfully performed in a simplified way. During the past several years, our center modified the traditional surgical techniques and applied them in a group of patients at high risk. The early and midterm results were encouraging and both in-hospital and 30-day mortalities were dramatically reduced.

The primary objective of this study was to evaluate the safety and feasibility of our simplified redo isolated tricuspid valve surgery on the beating heart through a minimally invasive approach. The secondary objective was to summarize our early and midterm results of management of high-risk isolated tricuspid valve regurgitation.

\section{Patients and methods}

The study protocol was approved by the Ethics Committee for the Protection of Human Subjects at the Zhongshan Hospital, Fudan University, Shanghai, China. Individual patient informed consent was not required in this study. The study was designed as a retrospective, observational study.

\section{Patient demographic and clinical characteristics}

A total of 32 consecutive patients who had undergone redo isolated tricuspid valve operations with minimally invasive, beating-heart technique through a right lateral thoracotomy at Zhongshan Hospital, Fudan University, between June 2016 and April 2020, were reviewed retrospectively. The mean age of patients was $57.3 \pm 8.7$ years (range, 43-72 years), and 18 patients (56.3\%) were women.

The indications for tricuspid valve operation in our study were the presence of severe or more tricuspid regurgitation along with New York Heart Association (NYHA) class III-IV, or other signs of right-sided heart failure, including uncontrolled pedal edema, ascites, pleural effusion, biological valve failure, and mechanical tricuspid valve dysfunction. We did not include patients who had undergone median open thoracotomy for the first tricuspid valve operation $(n=6)$, median redo tricuspid valve surgery $(n=1)$, and minimally invasive first tricuspid valve surgery $(n=1)$.

All patients had medical history of previous cardiac operations, including first-time or second-time reoperative cardiac procedures. All previous cardiac operations were performed through a median sternotomy. Fifteen patients $(46.9 \%)$ had previously undergone a mitral valve replacement, followed by five patients (15.6\%) with mitral valve replacement and tricuspid valve repair, and nine patients (28.1\%) with replacement of mitral and aortic valves. Detailed surgical procedures performed in previous operations are summarized in Table 1.

Pathological characteristics of the tricuspid valve included annulus dilatation, leaflet restriction, and thickening associated with severe or more tricuspid regurgitation in the majority patients (30 patients, 93.8\%), whereas two patients displayed mechanical and biological valve failure after tricuspid valve replacement.

\section{Surgical technique}

Although techniques of minimally invasive tricuspid valve surgery have been already reported elsewhere, there are still many differences in the way it is conducted in our center. Following induction of anesthesia and intubation with a dual-lumen endotracheal tube, all patients were routinely placed with transesophageal echocardiography (TEE) and implanted with an endocardial temporary pacing lead through the right internal jugular vein by an anesthetist. Patients were then positioned supine with the right shoulder elevated to $30^{\circ}$. In our center, cardiopulmonary bypass was established by femoral platform. An oblique incision was made usually in the right groin to expose the femoral artery and vein for cannulation. Utilizing the Seldinger technique, the femoral artery was cannulated with a $16 \mathrm{Fr}$ to $20 \mathrm{Fr}$ arterial cannula (Edwards Lifesciences, or Medtronic, USA), and the femoral vein was cannulated with a $24 \mathrm{Fr}$ venous cannula (Edwards Lifesciences). TEE was used to facilitate the placement of the femoral venous cannula up to the superior vena cava.

Table 1 Surgical procedures performed in previous operations

\begin{tabular}{ll}
\hline Surgical procedures & All patients $(\boldsymbol{n}=32)$ \\
\hline MVR & $15(46.9 \%)$ \\
TVR & $1(3.1 \%)$ \\
MVR + TVRep & $5(15.6 \%)$ \\
MVR + TVR & $1(3.1 \%)$ \\
MVR + AVR & $9(28.1 \%)$ \\
CABG & $1(3.1 \%)$ \\
\hline
\end{tabular}

MVR Mitral valve replacement, TVR tricuspid valve replacement, TVRep tricuspid valve repair, $A V R$ aortic valve replacement, $C A B G$ coronary artery bypass grafting 
After initiation of cardiopulmonary bypass, vacuum assistance (10-15 $\left.\mathrm{cm} \mathrm{H}_{2} \mathrm{O}\right)$ was usually used to accomplish total drainage of the atrium. A 5-cm right anterolateral thoracotomy was performed over the fourth intercostal space. After the fourth intercostal space was entered, the lungs were deflated and the right atrium was identified by pushing on the atrial wall with long forceps. Pericardial and atrial tissues were usually bonded tightly as a result of previous operations. Therefore, the pericardium was not dissected from the right atrium, but incised directly. Additionally, an auxiliary operating hole was made around the main incision for continuous carbon dioxide insufflation and passing the traction lines. Using traction lines to expose the right atrium and keeping the balance between arterial inflow and venous drainage, the tricuspid valve was visualized, and replacement or repair was performed on the beating heart with good visual field. After the operation was completed, the original operating hole was used to place the thoracic drainage tube.

\section{Follow-up}

The follow-up was performed either by direct interviews in our outpatient department to evaluate patients' clinical status or by telephone contact with patients and/or family members. The midterm follow-up was $87.1 \%$ complete with a mean duration of $26.3 \pm 12.3$ months.

\section{Statistical analysis}

Surgical treatment technique was not randomized, but was rather determined by the best medical judgment for each individual case. Data were collected from chart reviews, and were entered into a dedicated Microsoft Excel table.

Categorical variables were represented as frequency distributions and single percentages. Values of continuous variables were expressed as a mean \pm standard deviation (SD). Results were considered statistically significant at a p-level below 0.05. All analyses were performed in IBM SPSS statistical package version 26.0 (IBM Corp., Armonk, NY, USA).

\section{Results}

Demographic and clinical characteristics of the patients are depicted in Table 2. Apart from one patient with opening restriction of the artificial mechanical tricuspid valve, all other cases had severe or even tornado tricuspid regurgitation. All patients had clinical manifestations of the right-sided heart failure, including repeated chest tightness and asthma, uncontrolled pedal edema, ascites, and pleural effusion. Their average EuroSCORE was $7.8 \pm 1.4$ and predicted mortality was nearly $11.2 \%$. Eleven patients belonged to the NYHA functional class IV and 21 patients to the class III. Left ventricular ejection fraction (EF) of patients was almost normal in $58.3 \pm 7.1 \%$. In contrast, right ventricular function
Table 2 Demographic and clinical characteristics of the patients

\begin{tabular}{ll}
\hline Variable & All Patients $(n=32)$ \\
\hline Age (years) & $57.4 \pm 8.7(43$ to 72$)$ \\
Female & $18(56.3 \%)$ \\
NYHA class & \\
$\quad$ I & 0 \\
$\quad$ II & 0 \\
$\quad$ II & $21(65.6 \%)$ \\
mean EuroSCORE & $11(34.4 \%)$ \\
LVEF & $7.8 \pm 1.4$ \\
TAPSE (mm) & $58.3 \pm 7.1 \%$ \\
Mean PAP (mmHg) & $15.1 \pm 2.8$ \\
Creatinine level (umol/L) & $37.7 \pm 11.3$ \\
Bilirubin level (umol/L) & $88.9 \pm 31.8$ \\
INR & $24.8 \pm 17.3$ \\
Thrombocytes (10^9) & $2.3 \pm 0.8$ \\
Atrial fibrillation & $135.1 \pm 55.9$ \\
Atrial flutter & $27(84.3 \%)$ \\
Hypertension & $2(6.3 \%)$ \\
Diabetes mellitus & $10(31.3 \%)$ \\
Preoperative permanent pacemaker & $5(15.6 \%)$ \\
\hline
\end{tabular}

NYHA New York Heart Association, EuroSCORE European system for cardiac operative risk evaluation, LVEF left ventricular ejection fraction, TAPSE tricuspid annular plane systolic excusion, PAP pulmonary artery pressure, INR international standard ratio

deteriorated progressively due to severe tricuspid regurgitation, and tricuspid annular plane systolic excursion (TAPSE) was $15.1 \pm 2.8 \mathrm{~mm}$. Atrial fibrillation was recorded in 27 patients $(84.3 \%)$.

Table 3 describes the intraoperative course. All patients underwent minimally invasive beating-heart tricuspid valve replacement or repair (TVR/Rep) without

Table 3 Operative characteristics of the patients

\begin{tabular}{ll}
\hline Variable & All patients $(n=32)$ \\
\hline Functional TR & $25(78.1 \%)$ \\
Mechanical valve dysfunction & $1(3.1 \%)$ \\
Biological valve failure & $1(3.1 \%)$ \\
Etiology (previous TVRep) & $5(15.6 \%)$ \\
CPB time (min) & $81.5 \pm 29.0$ \\
TVRep & $2(6.2 \%)$ \\
TVR & $30(93.8 \%)$ \\
Conversion to sternotomy & 0 \\
Transfused RBC (units) & $0.5 \pm 1.3$ \\
Transfused Serum (ml) & $125.0 \pm 236.9$
\end{tabular}

$T R$ tricuspid regurgitation, $C P B$, cardiopulmonary bypass, TVR tricuspid valve replacement, TVRep tricuspid valve repair, $R B C$ red blood cell 
aortic clamping. There were no cases during the operation requiring urgent conversion to the median sternotomy. Thirty patients $(93.8 \%)$ had their tricuspid valve replaced, whereas two patients (6.2\%) had their tricuspid valve repaired. A biological prosthesis was used in all cases when the valve had to be replaced. The average valve size was $30.4 \pm 1.2 \mathrm{~mm}$. One patient underwent TVRep with semiflexible ring, and one patient underwent tricuspid valve reconstruction with calf pericardium. Average cardiopulmonary bypass time was $81.5 \pm 29.0 \mathrm{~min}$. Intraoperative exploration revealed severe tricuspid regurgitation in 25 patients was caused by enlarged tricuspid annulus and atrioventricular enlargement. A single patient had tricuspid valve limited opening due to artificial mechanical tricuspid valve thrombosis, one had a biological valve failure, while five patients showed organic damage of the valve texture due to previous tricuspid valve surgery. Nine patients (28.1\%) needed blood transfusions due to preoperative anemia, and received an average of $0.5 \pm 1.3$ units of red blood cell $(\mathrm{RBC})$ transfusion.

Postoperative course is described in Table 4. Both inhospital and overall thirty-day mortalities were $3.1 \%$. Overall in-hospital duration and ICU time were $13.6 \pm$ 7.6 days and $4.1 \pm 2.8$ days, respectively. Postoperative complications included prolonged ventilation, requiring ventilator-assisted support for more than $72 \mathrm{~h}$ in six patients (18.8\%). Three patients (9.4\%) developed acute renal insufficiency after operation and needed perioperative hemodialysis treatment. One patient (3.1\%) suffered permanent neurologic dysfunction, one $(3.1 \%)$ had a wound infection, and one required postoperative implantation of a permanent pacemaker because of permanent third-degree atrioventricular block. There were no complications such as perioperative myocardial infarction, reoperation for bleeding, mediastinal infection, or other complications. Thirty-one patients were discharged successfully.

Table 4 Postoperative outcomes of the patients

\begin{tabular}{ll}
\hline Variable & All patients $(n=32)$ \\
\hline Duration of ICU stay(d) & $4.1 \pm 2.8$ \\
Duration of hospital stay(d) & $13.6 \pm 7.6$ \\
Prolonged ventilation(n) & $6(18.8 \%)$ \\
Reoperation for bleeding & 0 \\
Postoperative neurologic event & $1(3.1 \%)$ \\
New-onset renal insufficiency requiring dialysis & $3(9.4 \%)$ \\
Wound infection & $1(3.1 \%)$ \\
In-hospital mortality & $1(3.1 \%)$ \\
30-d mortality & $1(3.1 \%)$ \\
Permanent III atrioventricular block & $1(3.1 \%)$ \\
\hline
\end{tabular}

ICU intensive care unit
Table 5 shows the preoperative functional NYHA class of the patients as well as their NYHA status during follow-up after TVR/Rep. The mean midterm follow-up period was $26.3 \pm 12.3$ months, and the follow-up was complete for 27 patients (87.1\%). Four patients were lost to follow-up after TVR or TVRep due to changed contact address. Cardiac function improved significantly in all 27 followed patients. Among them, 23 patients (85.2\%) significantly improved to NYHA class I compared with their preoperative status $(p=0.000)$. Three patients improved to NYHA class II compared with their preoperative status $(p=0.053)$. There were no deaths in patients who were followed. One patient underwent radiofrequency ablation for rapid atrial fibrillation. One patient received permanent pacemaker implantation for recurrent syncope due to bradycardia. No reoperations were necessary during the follow-up period.

\section{Discussion}

We routinely performed redo surgery using a median sternum split before June 2016 with mortality of up to $18.3 \%$ [7]. In the present study, we showed that by using simplified, minimally invasive, beating-heart technique, both the mortality and morbidity of redo isolated tricuspid valve surgery were greatly reduced. Most patients were discharged uneventfully. Our favorable early and midterm results are likely related not only to the minimally invasive access, which avoids extensive dissection and minimizes the risk of bleeding, but also to beatingheart technique, which decreases or eliminates ischemia reperfusion injury that follows standard maneuvers of aortic cross-clamping and clamp release. However, for patients undergoing reoperative surgery, the traditional median sternotomy approach is associated with a high risk of bleeding and risk of heart injury. In addition, obese and diabetic patients are particularly prone to sternal infection and sternal instability [13]. Although minimally invasive technique has been reported elsewhere [14-17], the procedure as conducted in our center includes many specific technical details. It is reasonable to expect that every technical detail contributes to the success of an operation. In our center, all patients were routinely implanted with endocardial temporary pacing lead through right internal jugular

Table 5 Preoperative and postoperative New York Heart Association (NYHA) classification

\begin{tabular}{llll}
\hline $\begin{array}{l}\text { NYHA } \\
\text { Classification }\end{array}$ & $\begin{array}{l}\text { Preoperative }(\boldsymbol{n}= \\
32)\end{array}$ & $\begin{array}{l}\text { To the present }(\boldsymbol{n}= \\
27)\end{array}$ & $\boldsymbol{p}$ value \\
\hline I & 0 & $23(85.2 \%)$ & 0.000 \\
II & 0 & $3(11.1 \%)$ & 0.053 \\
III & $21(65.6 \%)$ & $1(3.7 \%)$ & 0.000 \\
IV & $11(34.4 \%)$ & 0 & 0.001 \\
\hline
\end{tabular}


vein by the anesthetist before the operation, because the walking path of the conduction beam is very close to the operation area and nobody can surely avoid the thirddegree atrioventricular block for tricuspid valve replacement. To provide a bloodless working environment, some surgeons prefer using bicaval cannulation techniques with caval tapes or balloon $[10,18,19]$. We chose the "one incision, two cannulas" technique [20] for all of our cases. Femoral venous cannula was inserted up to the superior vena cava under the assistance of TEE. The positions of the superior and inferior vena cava were at a lower point compared with the tricuspid valve position. Therefore, there was no need to worry that a large amount of gas would enter the venous drainage line [21]. Meanwhile, a vacuum was also used to achieve a good venous drainage. By applying these techniques, we easily achieved a bloodless surgical field.

Some early reports showed a lower incidence of stroke with aortic versus femoral artery cannulation. The reason may be that peripheral cannulation increases the incidence of retrograde embolism from the descending thoracoabdominal aorta. However, femoral artery cannulation was routinely established for minimally invasive surgeries in our center, and stroke occurred in only one patient in the present series. Likewise, Lamelas evaluated all minimally invasive cardiac procedures that utilized femoral arterial cannulation over the past 6 years, and reported acceptably low incidences of stroke $(1.17 \%)$ and peripheral arterial trauma $(0.07 \%$; e.g., dissection, pseudoaneurysm, and fistula) [22]. Nifong et al. also reported low stroke risk with femoral artery cannulation [23]. Shann and Melnitchouk reported that the femoral artery cannulation is often used for procedures that utilize a right anterolateral minithoracotomy incision [24]. Overall, the main principle of minimally invasive surgery should leave the limited view for surgical procedures to the most degree by the establishment of peripheral CPB.

In our series, although most of redo tricuspid regurgitation was due to functional change, 93.8\% (30/32) of patients underwent tricuspid valve replacement instead of repair. Considering characteristics of our patients, such as younger mean age, previous cardiac surgery, chronic atrial fibrillation, and severe congestive heart failure (NYHA III-IV), we think that tricuspid valve replacement is the best option to avoid recurrent tricuspid regurgitation. We agree with Pfannmuller's opinion, and we also routinely use biological prostheses, regardless of a patient's age, even in patients with a previously implanted mechanical valve (regardless of whether it is in the aortic or mitral position, or both) to avoid trouble due to endocardial temporary pacing lead implantation and excessive anticoagulation mandatory for a mechanical prosthesis in a tricuspid position [25]. While some researchers reported that tricuspid valve replacement was always associated with significantly higher mortality $[1,16]$, others found no statistically significant differences in early and late outcomes between the isolated tricuspid valve repair and replacement surgeries [6]. However, one study reported a reduced mortality with the replacement surgery, which is in accordance with our results [3]. The choice of replacement or repair should be based on the surgeons' comprehensive judgment of the patient's overall state as well as personal experience.

\section{Study limitations}

There are several limitations of this study. First, it was only a retrospective, clinical observational trial in a single center, which has likely led to bias in patient selection and technique used. For final confirmation of our results, a prospective, multi-center study involving a larger sample size is required. Secondly, the small size of our series (only 32 patients) may influence generalizability of our findings. Additionally, although the early and midterm follow-up results are excellent, we do not yet have long-term followup data with our present technique. Finally, surgical treatment technique was not randomized, but rather was determined by the best medical judgment for each individual case. Therefore, we do not have an appropriate control group.

\section{Conclusions}

In this series of high-risk patients, redo tricuspid valve operation with a simplified, minimally invasive, beatingheart technique was both feasible and safe. The approach was associated with encouraging early and midterm results, showing low mortality and morbidity. Further studies are required to allow a direct comparison between our present technique and median sternotomy. Long-term data are also needed regarding the durability of these procedures.

\section{Abbreviations \\ CPB: Cardiopulmonary bypass; EuroSCORE: European system for cardiac operative risk evaluation; ICU: Intensive care unit; INR: International standard ratio; LVEF: Left ventricular ejection fraction; NYHA: New York Heart Association; PAP: Pulmonary artery pressure; RBC: Red blood cell; TAPSE: Tricuspid annular plane systolic excursion;; TR: Tricuspid regurgitation; TVR: Tricuspid valve replacement; TVRep: Tricuspid valve repair}

\section{Acknowledgements}

Not applicable.

\section{Authors' contributions}

SYL, KS and WCY carried out data collection, and drafted the manuscript. LMX participated in the operations and helped in drafting the manuscript. LLD and TH carried out data analysing and helped in revising the manuscript critically. CSW and SGY did the operations, conceived of the study, and helped in revising the manuscript critically. All authors read and approved the final manuscript. 


\section{Funding}

This study was supported by the National Natural Science Foundation of China (Grant no. 81501595), Youth Foundation of Zhongshan Hospital (Grant no. 2015ZSQN09), Talent Training Program Foundation for the Excellent Youth Supported by Zhongshan Hospital (Grant no. 2017ZSYQ24), Innovation Fund of Zhongshan Hospital (Grant no. 2017ZSCX05).

\section{Availability of data and materials}

Not applicable.

\section{Ethics approval and consent to participate}

The study protocol was approved by the Ethics Committee for the Protection of Human Subjects at the Zhongshan Hospital Fudan University (Shanghai, China). The study design was a retrospective observational study. Individual patient informed consent was not required in this study.

\section{Consent for publication}

All authors have read and approved the content and agree to submit it for consideration for publication in your journal.

\section{Competing interests}

The authors declare that they have no competing interests.

\section{Author details}

${ }^{1}$ Department of Cardiovascular Surgery, Zhongshan Hospital, Fudan University, The Shanghai Institute of Cardiovascular Diseases, No. 1609 Xietu Road, Xuhui District, Shanghai 200032, China. ${ }^{2}$ Department of Echocardiography, Zhongshan Hospital, Fudan University, Shanghai 200032, China.

Received: 19 March 2020 Accepted: 8 June 2020

Published online: 18 June 2020

\section{References}

1. Umehara N, Miyata H, Motomura N, Saito S, Yamazaki K. Surgical results of reoperative tricuspid surgery: analysis from the Japan cardiovascular surgery database. Interact Cardiovasc Thorac Surg. 2014;19(1):82-7.

2. Ricci D, Boffini M, Barbero C, El Qarra S, Marchetto G, Rinaldi M. Minimally invasive tricuspid valve surgery in patients at high risk. J Thorac Cardiovasc Surg. 2014;147(3):996-1001.

3. Buzzatti N, laci G, Taramasso M, Nisi T, Lapenna E, De Bonis M, Maisano F, Alfieri O. Long-term outcomes of tricuspid valve replacement after previous left-side heart surgery. Eur J Cardiothorac Surg. 2014;46(4):713-9 discussion 719.

4. Kilic A, Saha-Chaudhuri P, Rankin JS, Conte JV. Trends and outcomes of tricuspid valve surgery in North America: an analysis of more than 50,000 patients from the Society of Thoracic Surgeons database. Ann Thorac Surg 2013:96(5):1546-52 discussion 1552

5. Jeganathan R, Armstrong S, Al-Alao B, David T. The risk and outcomes of reoperative tricuspid valve surgery. Ann Thorac Surg. 2013:95(1):119-24.

6. Raikhelkar J, Lin HM, Neckman D, Afonso A, Scurlock C. Isolated tricuspid valve surgery: predictors of adverse outcome and survival. Heart Lung Circ. 2013;22(3):211-20.

7. Chen J, Abudupataer M, Hu K, Maimaiti A, Lu S, Wei L, Hong T, Wang C. Risk factors associated with perioperative morbidity and mortality following isolated tricuspid valve replacement. J Surg Res. 2018;221:224-31.

8. Casselman FP, La Meir M, Jeanmart H, Mazzarro E, Coddens J, Van Praet F, Wellens $F$, Vermeulen $Y$, Vanermen $H$. Endoscopic mitral and tricuspid valve surgery after previous cardiac surgery. Circulation. 2007:116(11 Suppl):1270-5.

9. Mihos CG, Pineda AM, Davila H, Larrauri-Reyes MC, Santana O. Combined mitral and tricuspid valve surgery performed via a right Minithoracotomy approach. Innovations (Phila). 2015;10(5):304-8.

10. Lee TC, Desai B, Glower DD. Results of 141 consecutive minimally invasive tricuspid valve operations: an 11-year experience. Ann Thorac Surg. 2009; 88(6):1845-50.

11. Minol JP, Boeken U, Weinreich T, Heimann M, Gramsch-Zabel H, Akhyari P, Kamiya $H$, Lichtenberg A. Isolated tricuspid valve surgery: a single institutional experience with the technique of minimally invasive surgery via right Minithoracotomy. Thorac Cardiovasc Surg. 2017;65(8):606-11.

12. Cao H, Zhou Q, Fan F, Xue Y, Pan J, Wang D. Right anterolateral thoracotomy: an attractive alternative to repeat sternotomy for high-risk patients undergoing reoperative mitral and tricuspid valve surgery. J Cardiothorac Surg. 2017;12(1):85.

13. Kypson AP, Glower DD. Minimally invasive tricuspid operation using port access. Ann Thorac Surg. 2002;74(1):43-5.

14. Pfannmüller B, Moz M, Misfeld M, Borger MA, Funkat AK, Garbade J, Mohr FW. Isolated tricuspid valve surgery in patients with previous cardiac surgery. J Thorac Cardiovasc Surg. 2013;146(4):841-7.

15. Urbandt P, Santana O, Mihos CG, Pineda AM. Joseph Lamelas. Minimally invasive approach for isolated tricuspid valve surgery. J Heart Valve Dis. 2014;23(6):783-7.

16. Seeburger J, Borger MA, Passage J, Misfeld M, Holzhey D, Noack T, Sauer M, Ender J, Mohr FW. Minimally invasive isolated tricuspid valve surgery. J Heart Valve Dis. 2010;19(2):189-92 discussion 193.

17. Botta L, Cannata A, Fratto P, Bruschi G, Trunfio S, Maneggia C, Martinelli L. The role of the minimally invasive beating heart technique in Reoperative valve surgery. J Card Surg. 2012;27(1):24-8.

18. Sansone F, Barbero C, Rinaldi M. Occlusion of both caval veins by an endovascular occluder. Heart Lung Circ. 2012;21(5):275-7.

19. Jiang Z, Mei J, Ding F, Shen S, Zhu J, Zhang J, Liu H, Yin H. Application of endovascular occlusion of both caval veins in minimally invasive isolated redo tricuspid surgery through right thoracotomy. Heart Lung Circ. 2014; 23(2):186-9.

20. Lu S, Wei L, Sun X, Wang C. One incision, two cannulations for minimally invasive aortic valve surgery. Int J Cardiol. 2015;182:97.

21. Peng $R$, Ba J, Wang $C$, Lai H, Hu K, Shi H. A new venous drainage technique in minimally invasive redo tricuspid surgery: vacuum-assist venous drainage via a single femoral venous cannula. Heart Lung Circ. 2017;26(2):201-4.

22. Lamelas J, Williams RF, Mawad M, LaPietra A. Complications associated with femoral Cannulation during minimally invasive cardiac surgery. Ann Thorac Surg. 2017;103(6):1927-32.

23. Nifong LW, Chitwood WR, Pappas PS, Smith CR, Argenziano M, Starnes VA, Shah PM. Robotic mitral valve surgery: a United States multicenter trial. J Thorac Cardiovasc Surg. 2005:129(6):1395-404.

24. Shann K, Melnitchouk S. Advances in perfusion techniques: minimally invasive procedures. Semin Cardiothorac Vasc Anesth. 2014;18(2):146-52.

25. Pfannmüller B, Misfeld M, Borger MA, Etz CD, Funkat AK, Garbade J, Mohr FW. Isolated reoperative minimally invasive tricuspid valve operations. Ann Thorac Surg. 2012;94(6):2005-10.

\section{Publisher's Note}

Springer Nature remains neutral with regard to jurisdictional claims in published maps and institutional affiliations.
Ready to submit your research? Choose BMC and benefit from:

- fast, convenient online submission

- thorough peer review by experienced researchers in your field

- rapid publication on acceptance

- support for research data, including large and complex data types

- gold Open Access which fosters wider collaboration and increased citations

- maximum visibility for your research: over $100 \mathrm{M}$ website views per year

At $\mathrm{BMC}$, research is always in progress.

Learn more biomedcentral.com/submissions 